\title{
DISSECTIONS OF A SIMPLEX
}

\section{BY G. L. ALEXANDERSON AND JOHN E. WETZEL}

Communicated by Paul T. Bateman, June 29, 1972

1. On each edge $e_{i}$ of a tetrahedron $T$, let $n_{i} \geqq 0$ points be selected and each joined by a triangular plane segment to the corresponding opposite edge of $T$. In [1] we proved that the number $C$ of disjoint cells formed inside $T$ satisfies

$$
C \leqq 1+\sigma_{1}+\sigma_{2}+\sigma_{3}^{*},
$$

where $\sigma_{1}$ is the sum of the six $n_{i}, \sigma_{2}$ is the sum of the 15 products $n_{i} n_{j}$ with $1 \leqq i<j \leqq 6$, and $\sigma_{3}^{*}$ is the sum of the 16 products $n_{i} n_{j} n_{k}$ for which the corresponding edges do not form a triangular path on $T$ and $1 \leqq i<j<k \leqq 6$. The equality holds if and only if the triangular plane segments are in "interior general position," i.e., no three meet in an interior line segment and no four meet in an interior point of $T$.

In this note we announce the analogous result for a dissected simplex in $E^{d}$.

2. Let $T^{d}$ be a simplex in $E^{d}$, the convex hull of $d+1$ independent vertices $V_{0}, V_{1}, \ldots, V_{d}$, and denote by $e_{i j}$ the edge of $T^{d}$ that joins $V_{i}$ and $V_{j}$. On each edge $e_{i j}$ let $n_{i j} \geqq 0$ points (not vertices) be selected. With the $d-1$ opposite vertices of $T^{d}$, each such point determines a $(d-1)$-simplex, which dissects $T^{d}$. Let $H$ be the number of nonoverlapping $(d-1)$ polytopes into which the $\sum n_{i j}$ dissecting simplices divide $T^{d}$.

For each $m=1,2, \ldots, d$, let

$$
\sigma_{m}^{*}=\sum^{*} n_{i_{1} j_{1}} n_{i_{2} j_{2}} \cdots n_{i_{m} j_{m}},
$$

the sum being extended over all $m$-sets of edges $e_{i_{1} j_{1}}, e_{i_{2} j_{2}}, \ldots, e_{i_{m} j_{m}}$ no subcollection of which forms a closed path on $T^{d}$; and let $H_{d}=1+\sum_{m=1}^{d} \sigma_{m}^{*}$.

Our result is that $H \leqq H_{d}$, and the equality holds if and only if the dissecting simplices are in "interior general position," i.e., for each $k$ with $2 \leqq k \leqq d$, no $k+1$ dissecting simplices meet in a $(d-k)$-simplex.

The proof is by induction on the dimension $d$. Let $\boldsymbol{n}=\left\langle n_{i j}\right\rangle$ be the $\left(\begin{array}{c}d+1 \\ 2\end{array}\right)$-vector of data for $T^{d}$, the components being listed in lexicographic order. Let $T_{b}$ be the "base" $(d-1)$-simplex, determined by the vertices $V_{0}, V_{1}, \ldots, V_{d-1}$, and let $\boldsymbol{n}_{b}$ be the $\left(\begin{array}{l}d \\ 2\end{array}\right)$-vector of data for $T_{b}$, with the components in lexicographic order. Let $s_{i j}$ be the $\left(\begin{array}{l}d \\ 2\end{array}\right)$-vector having 1 in the

AMS (MOS) subject classifications (1970). Primary 50B30.

Copyright (C) American Mathematical Society 1973 
ijth position and 0 elsewhere.

We show on geometric grounds that the maximum number $H_{d}$ of nonoverlapping $(d-1)$-polytopes formed in $T^{d}$ satisfies the recursion

$$
\begin{aligned}
H_{1}\left(\left\langle n_{01}\right\rangle\right) & =1+n_{01}, \\
H_{d}(\boldsymbol{n}) & =H_{d-1}\left(\boldsymbol{n}_{b}\right)+n_{0 d} H_{d-1}\left(\boldsymbol{n}_{b}\right)+\sum_{m=1}^{d-1} n_{m d} H_{d-1}\left(\boldsymbol{n}_{b}+\sum_{j=0}^{m-1} n_{j d} \boldsymbol{s}_{j m}\right)
\end{aligned}
$$

and then verify that the sequence $\left\{H_{d}\right\}$ defined above is the only solution of this recursion. Details and some related results will be published elsewhere.

\section{REFERENCE}

1. G. L. Alexanderson and John E. Wetzel, Dissections of a tetrahedron, J. Combinatorial Theory 11 (1971), 58-66.

Department of Mathematics, University of Santa Clara, Santa Clara, California 95053 (current address of G. L. Alexanderson)

Current address (J. E. Wetzel): Department of Mathematics, University of Illinois, Urbana, Illinois 61801 